\title{
EV-Associated MMP9 in High-Grade Serous Ovarian Cancer Is Preferentially Localized to Annexin V-Binding EVs
}

\author{
Agnes T. Reiner, ${ }^{1,2,3}$ Sisareuth Tan, ${ }^{4}$ Christiane Agreiter, ${ }^{3}$ Katharina Auer, ${ }^{3}$ \\ Anna Bachmayr-Heyda, ${ }^{3}$ Stefanie Aust, ${ }^{3}$ Nina Pecha, ${ }^{3}$ Mattias Mandorfer, ${ }^{5}$ Dietmar Pils, ${ }^{6,7}$ \\ Alain R. Brisson, ${ }^{4}$ Robert Zeillinger, ${ }^{3}$ and Sai Kiang Lim $^{2}$ \\ ${ }^{1}$ BioSensor Technologies, AIT-Austrian Institute of Technology GmbH, Muthgasse 11, 1190 Vienna, Austria \\ ${ }^{2}$ Institute of Medical Biology, A*STAR, 8A Biomedical Grove, No. 05-05 Immunos, Singapore 138648 \\ ${ }^{3}$ Molecular Oncology Group, Department of Obstetrics and Gynecology, Medical University of Vienna, Waehringer Guertel 18-20, \\ 1090 Vienna, Austria \\ ${ }^{4}$ Extracellular Vesicles and Membrane Repair, UMR-5248-CBMN, CNRS-University of Bordeaux-IPB, Allée Geoffroy Saint-Hilaire, \\ 33600 Pessac, France \\ ${ }^{5}$ Division of Gastroenterology and Hepatology, Department of Internal Medicine III, Medical University of Vienna, Waehringer \\ Guertel 18-20, 1090 Vienna, Austria \\ ${ }^{6}$ Section for Clinical Biometrics, Center for Medical Statistics, Informatics, and Intelligent Systems (CeMSIIS), Medical University of \\ Vienna, Waehringer Guertel 18-20, 1090 Vienna, Austria \\ ${ }^{7}$ Department of Surgery, Medical University of Vienna, Waehringer Guertel 18-20, 1090 Vienna, Austria
}

Correspondence should be addressed to Sai Kiang Lim; saikiang.lim@imb.a-star.edu.sg

Received 24 November 2016; Revised 24 January 2017; Accepted 5 April 2017; Published 18 May 2017

Academic Editor: Alvaro González

Copyright @ 2017 Agnes T. Reiner et al. This is an open access article distributed under the Creative Commons Attribution License, which permits unrestricted use, distribution, and reproduction in any medium, provided the original work is properly cited.

High-grade serous ovarian cancer (HGSOC) is the most aggressive type of ovarian cancer and is responsible for most deaths caused by gynecological cancers. Numerous candidate biomarkers were identified for this disease in the last decades, but most were not sensitive or specific enough for clinical applications. Hence, new biomarkers for HGSOC are urgently required. This study aimed to identify new markers by isolating different extracellular vesicle (EV) types from the ascites of ovarian cancer patients according to their affinities for lipid-binding proteins and analyzing their protein cargo. This approach circumvents the low signal-to-noise ratio when using biological fluids for biomarker discovery and the issue of contamination by large non-EV complexes. We isolated and analyzed three distinct EV populations from the ascites of patients with ovarian cancer or cirrhosis and observed that Annexin V-binding EVs have higher levels of matrix metalloproteinase 9 in malignant compared to portal-hypertensive ascites. As this protein was not detected in other EV populations, this study validates our approach of using different EV types for optimal biomarker discovery. Furthermore, MMP9 in Annexin V-binding EVs could be a HGSOC biomarker with enhanced specificity, because its identification requires detection of two distinct components, that is, lipid and protein.

\section{Introduction}

Ovarian cancer is the deadliest among gynecologic malignancies with a 5-year survival rate after diagnosis of $27 \%$ [1]. This poor prognosis is mostly due to the asymptomatic disease progression, resulting in diagnosis at advanced stages [2] and to the lack of reliable biomarkers. Over the last few years, extracellular vesicles (EVs) have gained a lot of interest as potential sources of biomarkers in many diseases including ovarian cancer, because the secreted vesicles and their cargo resemble their cell of origin and, additionally, EVs can be easily accessed and isolated from any of our bodily fluids [3]. The collection of bodily fluids is generally less invasive than conventional tissue biopsy. In several studies of ovarian 
cancer EVs, cancer-derived vesicles have been identified in blood plasma, serum, and ascites fluid of patients and they presented a unique set of cargo proteins and RNAs with great potential as new diagnostic, prognostic, and predictive biomarkers $[4,5]$.

However, the current methods to isolate EVs from body fluids for protein biomarkers are particularly challenging for several reasons. First, the proteome of most bodily fluids is complex and protein concentrations have a dynamic range that exceeds $10^{10}$. For example, the proteome of plasma or serum is dominated by several high-abundance proteins. It is estimated that of the approximate 10,000 proteins in the serum, only 21 proteins (namely, albumin; IgG; transferrin; haptoglobin; transthyretin; $\alpha 2$-macroglobulin; $\alpha 1$-antitrypsin; $\alpha 1$-acid glycoprotein; hemopexin; apolipoprotein A1 and $\mathrm{B}$; AGP; lipoprotein A; factor $\mathrm{H}$; ceruloplasmin; complements C4, C9, C19, and C8; and prealbumin) constitute $>99 \%$ of the serum protein mass [6]. As such, any technique that aims to isolate candidate biomarkers will have to isolate the remaining $1 \%$ from the bulk of high-abundance proteins. Hence, common EV isolation methods based on biophysical parameters such as high-speed centrifugation or filtration would inevitably be contaminated with the high-abundance proteins $[7,8]$. Second, in addition to proteins, many bodily fluids contain large lipoprotein complexes that are similar in size and density to EVs and thus are often copurified and mistaken as EVs by common analysis methods $[9,10]$. And third, individual classes of EVs, for instance, exosomes or microvesicles, are very difficult to discriminate, because they overlap not only in their physical characteristics like size or density but also in their cargo molecules [11]. Consequently, this lack of distinguishing criteria makes the isolation of pure vesicle populations almost impossible with currently used methods. Additionally, recent publications suggest that even within the earlier defined classes of EVs, there are more subclasses of vesicles with distinct cargo and functionality [12] adding another layer of complexity.

To overcome some of these obstacles, a new strategy for vesicle isolation using proteins with high specific binding affinity for membrane lipids has been proposed. In this approach, vesicles are isolated based on their membrane lipid composition, which resolves the problem of protein contamination and introduces a new way of classifying vesicle populations. The proteins used for isolation are Annexin V (AV), Cholera Toxin B-chain (CTB), and Shiga Toxin B-chain (STB), which have high specific binding affinity for phosphatidylserine, ganglioside GM1, and globotriaosylceramide, respectively. By combining these lipid-binding ligands with magnetic particles, distinct populations of EVs that can be bound by each of the lipid ligands were purified and characterized from conditioned medium of mesenchymal stem cells [13]. This lipid-based isolation approach was also used to successfully identify protein biomarkers in EVs isolated from human plasma for the diagnosis of preeclampsia [14].

The aim of this study was to examine the potential of ascitic fluid-derived EVs as markers for ovarian cancer using this new vesicle isolation method based on specific binding of lipids present in the vesicle membrane. First, the isolation method was tested for successful isolation of EVs from ascites and then the protein cargo of different EV populations isolated from the ascitic fluid of patients with ovarian cancer and cirrhosis was analyzed in regard to the presence of cancer-associated molecules.

\section{Material and Methods}

2.1. Patient Samples. The ascites of patients with ovarian cancer or cirrhosis was collected at the Medical University of Vienna (Austria). Patients with ovarian cancer recruited in the time from September 2013 to January 2015 were included in the study, if ascites was present and no treatment was performed before sample collection. Patients with cirrhosis were recruited between July and August 2015 and were excluded, if cirrhosis was due to hepatitis C. All patients signed an informed consent before sample collection. This study was approved by the ethical review board of the Medical University of Vienna (number 793/2011). The ascites samples of ovarian cancer patients were collected at the first presentation of the disease, before therapy or during debulking surgery.

After collection, the ascitic fluid was centrifuged at $1000 \mathrm{~g}$ for $10 \mathrm{~min}$ and the supernatant was centrifuged a second time at $3000 \mathrm{~g}$ for $10 \mathrm{~min}$ to remove remaining cells and cell debris. Subsequently, the supernatant was filtered through a $0.22 \mu \mathrm{m}$ filter (Merck Millipore, USA), aliquoted, and stored at $-80^{\circ} \mathrm{C}$ for further analysis.

2.2. EV Isolation with Lipid-Binding Ligands. The EV isolation procedure was performed as described earlier [13]. Briefly, $1.5 \mathrm{ng}$ of biotinylated lipid-binding ligand were used per $\mu \mathrm{l}$ of ascites. The ligands AV, CTB, and STB were mixed with ascites in binding buffer (10 mM HEPES, $2.5 \mathrm{mM} \mathrm{CaCl}_{2}$, $140 \mathrm{mM} \mathrm{NaCl}$, PBS pH 7.4) and incubated for 30 minutes at room temperature (RT) with shaking. Then, $0.1 \mu \mathrm{l}$ per $\mu \mathrm{l}$ ascites starting volume of prewashed Dynabeads M280 Streptavidin (Thermo Fisher Scientific, USA) were added to each reaction mix and incubated again for 30 minutes at RT with shaking. The beads were separated from the liquid with a magnet, the supernatant was discarded, and the beads were washed twice with PBS. Finally, the bead bound fraction was resuspended in PBS or lysis buffer depending on the subsequent analysis method. The starting volume of ascitic fluid for EV isolation was adjusted for each analysis method and is stated in the respective sections. As a negative control for unspecific binding to the magnetic beads, the same isolation procedure was performed without an addition of a lipidbinding ligand.

2.3. Western Blot. Standard western blot analysis was performed by denaturing the EV samples isolated from $250 \mu \mathrm{l}$ of ascites and loading them onto $4-12 \%$ NuPAGE Bis-Tris Gels in reducing conditions (Thermo Fisher Scientific, USA). After gel electrophoresis, the proteins were transferred to a nitrocellulose membrane. The membrane was then probed with a primary antibody followed by a HRPconjugated secondary antibody. The primary antibody was detected using a chemiluminescent HRP-substrate (Super Signal West Solution, Thermo Fisher Scientific, USA). The 
chemiluminescent signals were analyzed with Image Lab software (Bio-Rad Laboratories, USA). The band intensities of each individually analyzed protein were quantified by subtracting the background signal and normalizing to the corresponding $\beta$-actin band intensity. Results were given as the protein to $\beta$-actin ratio. The primary antibodies were mouse antibodies against the human proteins $\beta$ actin (C4), $\beta$-catenin (E-5), CD71 (H68.4), CD9 (C-4), Alix (1A12), CD59 (H-7), EpCAM (0.N.276) (Santa Cruz Biotechnology Inc., USA), CD63 (H5C6) (BD Biosciences, USA), and FN1-EDA (IST-9) (abcam PLC, UK). All primary antibodies were used in a 1:50 dilution; only antiFN1-EDA was diluted 1:500. The secondary antibody was a goat anti-mouse IgG-HRP conjugate (sc-2031, Santa Cruz Biotechnology Inc., USA) and diluted 1:1250.

2.4. Zymography and MMP9 ELISA. The presence of gelatinases in the EV isolates was analyzed using Novex Zymogram Gels (Thermo Fisher Scientific, USA) according to the manufacturer's instructions. The EV isolations from $500 \mu \mathrm{l}$ ascites and $1 \mu \mathrm{l}$ of ascites without isolation were diluted in the loading buffer and resolved on a 10\% zymogram (gelatin) gel in nonreducing conditions. After protein renaturation and developing the gel over night at $37^{\circ} \mathrm{C}$, the gel was stained with Coomassie Blue staining solution ( $40 \mathrm{vol} \%$ methanol, 7 vol\% acetic acid, and $0.25 \mathrm{mg} / \mathrm{ml}$ Brilliant Blue R (all Sigma Aldrich, USA), in water) for $30 \mathrm{~min}$ at $80^{\circ} \mathrm{C}$ and then for 4 hours at RT with agitation. Then, the gel was washed in destaining solution ( $40 \mathrm{vol} \%$ methanol, $7 \mathrm{vol} \%$ acetic acid, in water) for $10 \mathrm{~min}$ at $80^{\circ} \mathrm{C}$ and RT alternately.

The obtained images were analyzed with Image Lab software (Bio-Rad Laboratories, USA). The band intensities were quantified by extracting the background-corrected volume density and normalized to the number of pixels of the analyzed band. For quantification of MMP9-levels, only the $92 \mathrm{kDa}$ band was analyzed.

MMP9-levels of ascites or EV isolates were quantified by ELISA. The samples were lysed with the Mammalian Cell Extraction Kit (BioVision, USA) and subsequently analyzed with the Quantikine ELISA human MMP-9 kit (R\&D, USA) that were both performed according to the manufacturer's instructions, except for the sample incubation conditions in the ELISA that were changed to overnight at $4^{\circ} \mathrm{C}$.

2.5. Cryoelectron Microscopy and AV Gold Labeling. For cryoelectron microscopy (EM) experiments, ascites samples were labeled with $4 \mathrm{~nm}$ gold nanoparticles (NP) conjugated to $\mathrm{AV}$ as follows. An $8 \mu \mathrm{l}$ aliquot of ascites was mixed with $1 \mu \mathrm{l} 20 \mathrm{mM} \mathrm{CaCl}_{2}$ and $1 \mu \mathrm{l} 1-4 \times 10^{16} \mathrm{NP} / \mathrm{L}$ AV-gold-NP and incubated for $15 \mathrm{~min}$. Then, $4 \mu \mathrm{l}$ were deposited on an EM grid coated with a perforated carbon film and quickly frozen in liquid ethane. EM grids were mounted on a Gatan 626 cryoholder and transferred into a Tecnai F20 (FEI) microscope operated at $200 \mathrm{kV}$. Images were recorded with an USC1000-SSCCD camera (Gatan). For the synthesis of AV-gold-NPs, refer to Arraud et al. [15].

2.6. Statistical Analysis. The statistical analysis was performed in R. The 2-sided, unpaired Mann-Whitney-
TABLE 1: Clinical characteristics of ovarian cancer patients; na = not available; $*$ indicates that these samples were only used in the pool, because the detailed clinical characteristics were not available.

\begin{tabular}{lcccc}
\hline $\begin{array}{l}\text { Patient } \\
\text { number }\end{array}$ & $\begin{array}{c}\text { Histological } \\
\text { type }\end{array}$ & $\begin{array}{c}\text { Age at } \\
\text { diagnosis }\end{array}$ & $\begin{array}{c}\text { FIGO stage } \\
\text { at diagnosis }\end{array}$ & $\begin{array}{c}\text { Grade at } \\
\text { diagnosis }\end{array}$ \\
\hline 1 & Carcinosarcoma & na & IIIC & 3 \\
2 & Serous papillary & 60 & IV & 3 \\
3 & Serous papillary & 52 & IV & 3 \\
4 & Serous papillary & 46 & IIIC & 3 \\
5 & Carcinosarcoma & na & IIIC & 3 \\
6 & Serous papillary & 67 & IV & 2 \\
7 & Serous papillary & 83 & na & 2 \\
8 & Serous papillary & 60 & IV & 2 \\
$9 *$ & na & na & na & na \\
10 & Serous papillary & 39 & IV & 3 \\
11 & Serous papillary & 70 & na & 3 \\
$12^{*}$ & na & na & na & na \\
13 & Serous papillary & 73 & IIIC & 3 \\
14 & Serous papillary & 63 & IIIC & 2 \\
15 & Serous papillary & 60 & IIIC & 3 \\
16 & Serous papillary & 62 & IIIC & 3 \\
17 & Clear cell & 61 & IIIC & 3 \\
\hline
\end{tabular}

Wilcoxon test was used for the comparison of MMP9 levels measured by zymography or ELISA between different patient groups. The correlation between zymography and ELISA results was calculated with the Spearman method, and only the range from 0 to $100 \mathrm{pg}$ of MMP9 was used. The correlation coefficient (rho), as well as $p$ values, was given as results, and $p$ values $\leq 0.05$ were considered statistically significant.

\section{Results and Discussion}

3.1. Patient Cohort. The patient cohort comprised of 17 patients with ovarian cancer and 10 with cirrhosis, respectively. The former had a median age of 61 years (39-83 yrs) at diagnosis and recruitment to the study. The later had a median age of 66 years (53-77 yrs). The clinicopathological characteristics of the ovarian cancers-histological type, FIGO stage, and grade-were assessed by a pathologist after a routine surgical removal of the cancer tissue. All clinical details of the ovarian cancer patients are given in Table 1.

3.2. Isolation Method Testing. In this study, EVs were isolated by incubating the ascites fluid with biotinylated lipid-binding protein ligands AV, CTB, and STB. Vesicles bound by these biotinylated ligands were extracted with streptavidin-coated magnetic beads and thus could be separated from proteins or other vesicles present in the sample. As this method was only used for EV isolation from conditioned cell culture medium and plasma before $[13,14]$, we determined if these ligands could also isolate EVs from ascitic fluid by examining the isolates for the presence of tetraspanins CD9 and CD63, the transferrin receptor or CD71, and a cytosolic protein, Alix. All these proteins are associated with EVs and generally used as EV markers [3]. $\beta$-actin was used as a loading control 

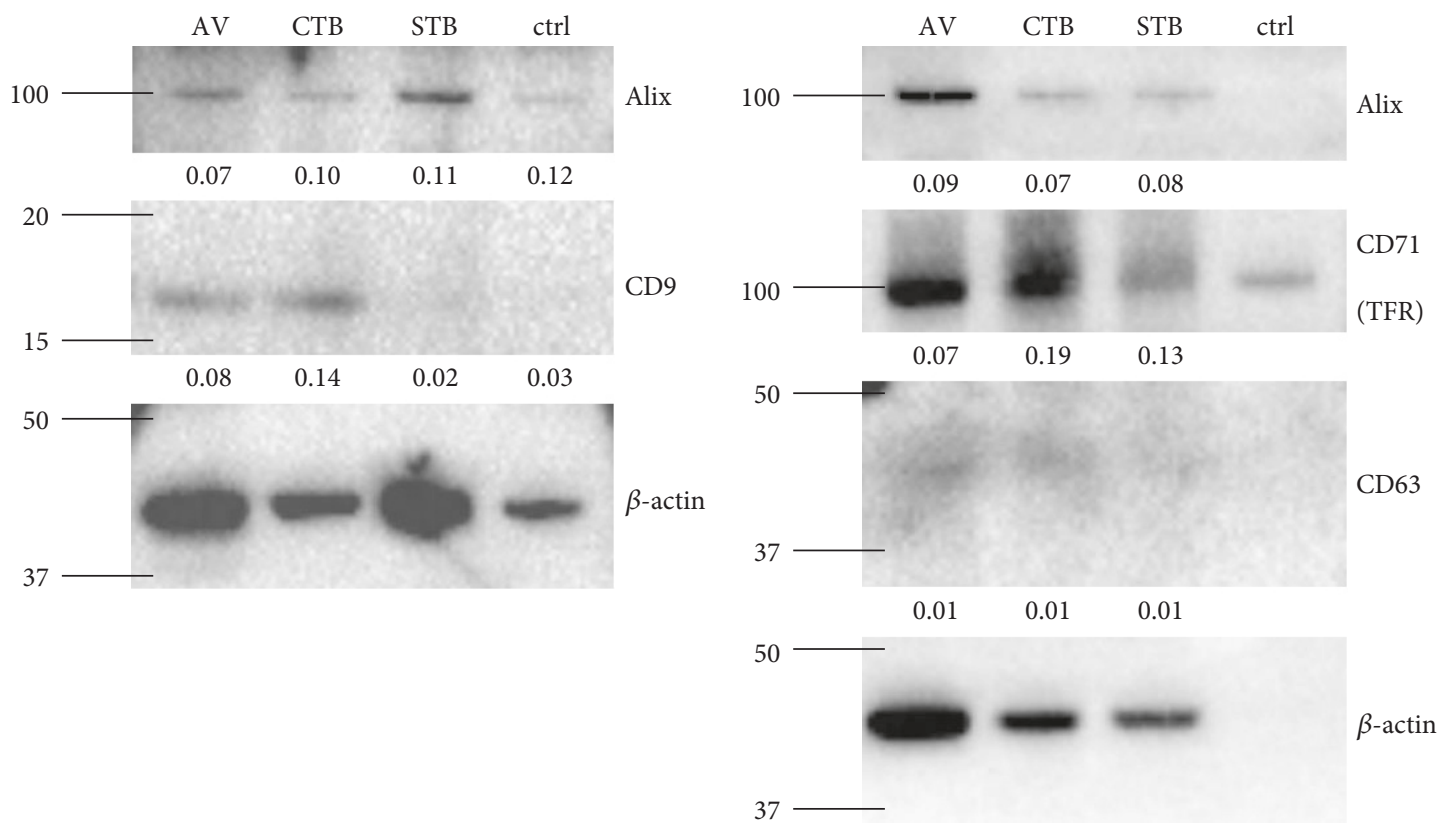

(a)

(b)

Figure 1: EV isolation from ascites. EVs were isolated from ascites with the ligands AV (lane 1), CTB (lane 2), and STB (lane 3 ) and analyzed by western blotting. As a negative control, no ligand was added (lane 4). $250 \mu \mathrm{l}$ of pooled ascites from 6 (a) or 16 (b) ovarian cancer patients were used as starting material. The tetraspanin proteins CD9 and CD63, the transferrin receptor (CD71), and the cytosolic protein Alix were examined, and $\beta$-actin was used as a loading control. The normalized band intensities of the analyzed proteins are given below each band. The molecular weight markers are indicated on the left side of the images in $\mathrm{kDa}$.

and for band intensity normalization. Due to limited sample volumes, this analysis was performed using two different pools of ascites, one pooled from 6 cancer patients (Figure 1(a)) and the other pooled from 16 cancer patients (Figure 1(b)). As shown in Figure 1, CD9 and CD63 were detected only in the AV- and CTB-binding EVs from ascites. CD71 and Alix were detected in all three vesicle populations. As a negative control for unspecific binding of ascites proteins to the magnetic beads, the same isolation procedure was performed without the addition of a ligand (Figure 1, lane 4). Even though some unspecific binding to the magnetic beads occurred, especially for $\beta$-actin and CD71, the level of the proteins in the negative control was very low compared to that in the EV isolations, suggesting specific enrichment of the tested proteins in the isolations. As CD9 and CD63 are tetraspanins with 4 membrane domains, their presence in the isolates were definitive evidence that all three isolates were lipid membrane vesicles. The different distribution profile of the four proteins relative to $\beta$-actin among the three EV isolates further suggested that each of the three EV isolates was unique. For example, the absence of CD9 in STB-binding EVs showed clearly that these EVs are different from both CTB-binding and AV-binding EVs [13]. Taking these results together, $\mathrm{AV}, \mathrm{CTB}$, and STB are capable of isolating unique $\mathrm{EV}$ populations from ascites.

3.3. Cancer-Marker Search. Next, AV-, CTB-, and STBbinding vesicles isolated from pooled ovarian cancer ascites or from pooled portal-hypertensive ascites were probed for the presence of cancer markers. The rationale for using ascitic fluid instead of plasma for the discovery of EVassociated ovarian cancer biomarkers is that ascites, being in direct contact with the cancer, is more likely to carry cancer biomarkers [1, 16-19]. Once candidate EV-associated ovarian cancer biomarkers are identified, we will next determine if these ascites EV-associated candidate biomarkers can be found in plasma as well. This strategy will enhance the chance of identifying ovarian cancer biomarkers where the signal-to-noise ratio will be higher than that in the plasma, where the vast majority of EVs are derived from blood cells. The following cancer-associated proteins were selected for analysis in the EV isolations based on literature search: cellular fibronectin (FN1-EDA), protectin (CD59), epithelial cell adhesion molecule (EpCAM), $\beta$-catenin, and gelatinases matrix metalloproteinase 2 and 9 (MMP2/9).

FN1-EDA, also called cellular fibronectin, is a splice variant of fibronectin and an important component of the extracellular matrix. It is highly expressed during embryogenic development but barely found in adult tissues in contrast to the soluble fibronectin isotype, which is constantly produced by hepatocytes and generally present in plasma [20]. FN1EDA was found in a variety of cancer tissues [21] and plays an important role in angiogenesis and cell migration [20] which are two processes crucial for carcinogenesis and metastasis. This protein was detected in all three EV types isolated from the ascites of patients with ovarian cancer and was highly elevated in AV- and STB-EVs of the cirrhosis patients (Figure 2). The elevated level of FN1-EDA in EVs of patients with cirrhosis is consistent with that of the previous report of its important role in experimental hepatic 


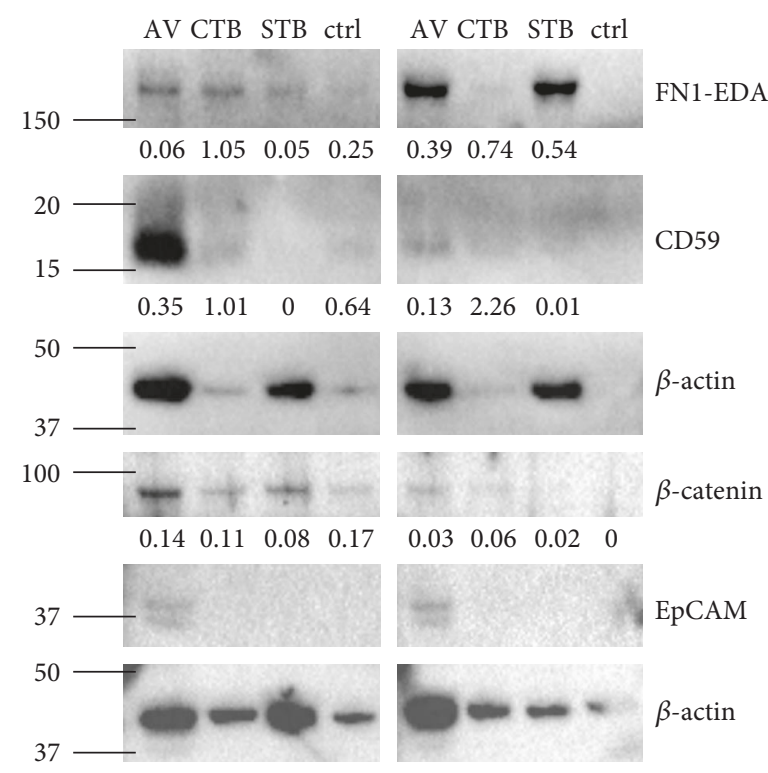

(a)

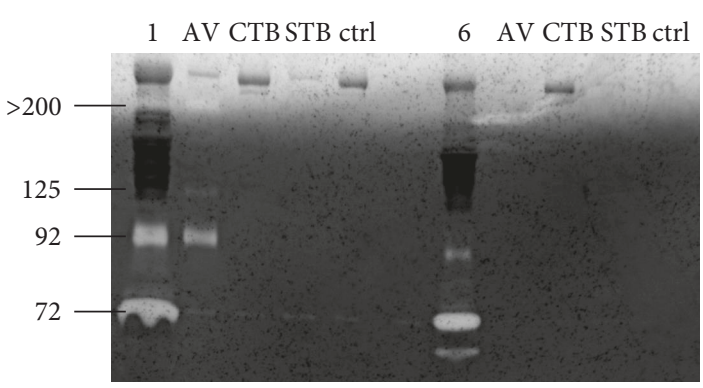

(b)

FIGURE 2: Cancer proteins in EV isolations. (a) EVs isolated from $250 \mu \mathrm{l}$ of pooled ascites from 6 ovarian cancer patients (lanes 1-4) or from 5 patients with cirrhosis (lanes 5-8) were analyzed by western blot. As before the ligands AV, CTB, and STB were used for isolation, no ligand was added as a negative control. The protein cellular fibronectin (FN1-EDA), CD59, $\beta$-catenin, and epithelial cell adhesion molecule (EpCAM) were examined, and $\beta$-actin was used as loading control. The normalized band intensities of the analyzed proteins are given below each band. (b) EVs isolated from $500 \mu \mathrm{l}$ of the same samples as described for (a) were analyzed by zymography. The left part of the image (lanes 1-5) shows the ovarian cancer samples and the right part the cirrhosis samples (lanes $6-10$ ). In lanes 1 and $6,1 \mu l$ of the pooled ascites samples were analyzed. The molecular weight markers are indicated on the left side of the images in $\mathrm{kDa}$.

fibrosis [22]. However, this observation eliminated the utility of FN1-EDA as an ovarian cancer marker.

CD59 is a GPI-anchored membrane protein that is expressed on the surface of cells to protect them from complement-mediated cell lysis by inhibiting the assembly of the membrane attack complex. Many types of cancer, including ovarian cancer, express CD59 to escape the immune system [23]. Furthermore, CD59 has been found on EVs which enabled them to protect themselves and other cells from complement attack [24-26]. Interestingly, it was previously reported that CD59 is not only present in the ascites of ovarian cancer patients but is also associated with the phospholipid fraction [27]. Consistent with this, we delete detected CD59 in EVs from ovarian cancer ascites and we also found that this protein was localized to AV-binding EVs (Figure 2, lane 1). Furthermore, these AV-binding EVs carried more CD59 than AV-binding EVs from portalhypertensive ascites (lane 5) after correcting for $\beta$-actin, making CD59 a potential biomarker for ovarian cancer.

Among the recently identified EV-based biomarkers for the diagnosis of ovarian cancer, EpCAM is one of the most intensively studied proteins. Several studies showed that the number of EpCAM-positive EVs in body fluids increases with disease progression [28-32]. However, in our EV isolations, EpCAM was not detectable (Figure 2). This could be due to the different isolation procedures used in the different studies, which result in different vesicle populations being analyzed, as well as highly variable contamination levels.

A well known protein which is overexpressed in many cancers is $\beta$-catenin. It acts via the $\mathrm{Wnt} / \beta$-catenin signaling pathway promoting cell growth and proliferation [33]. A recent study showed that $\beta$-catenin can be transferred between cancer cells via EVs and promotes cell motility and proliferation in the recipient cells [34]. This protein was identified in all vesicle populations from ovarian cancer ascites (Figure 2, lanes 1 to 3 ). Although the ratio of $\beta$-catenin to $\beta$-actin was higher in each of the three EVs in ovarian cancer ascites than those in the portal-hypertensive ascites, this ratio was similar to that in the no-ligand control suggesting that the signal is likely to be nonspecific.

3.4. MMP9 Cargo of AV-Binding EVs. The gelatinases MMP2 and MMP9 have crucial functions in cancer progression and have been identified in ovarian cancer tissues, ascites, and patient's circulation [35-37]. Using zymography in which the presence of both gelatinases in their active and inactive proform can be examined, we observed that relative to the portal-hypertensive ascites, the pooled ascitic fluid of ovarian cancer patients had a higher level of pro-MMP2 and proMMP9 with the molecular weights of $72 \mathrm{kDa}$ and $92 \mathrm{kDa}$, respectively. Additionally, the pooled portal-hypertensive ascites had the $60 \mathrm{kDa}$ active MMP2 which was not detected in ovarian cancer ascites (Figure 2(b), lane 1 versus 6). The higher MMP-levels in malignant ascites are generally consistent with the often reported higher expression and secretion of MMPs in the cancer microenvironment [38].

MMPs have already been reported to be present in EVs from ovarian cancer ascites and had been associated with a proinvasive effect on ovarian cancer cells [30, 39]. Here, we not only confirmed the presence of MMP9 in EVs but we also 


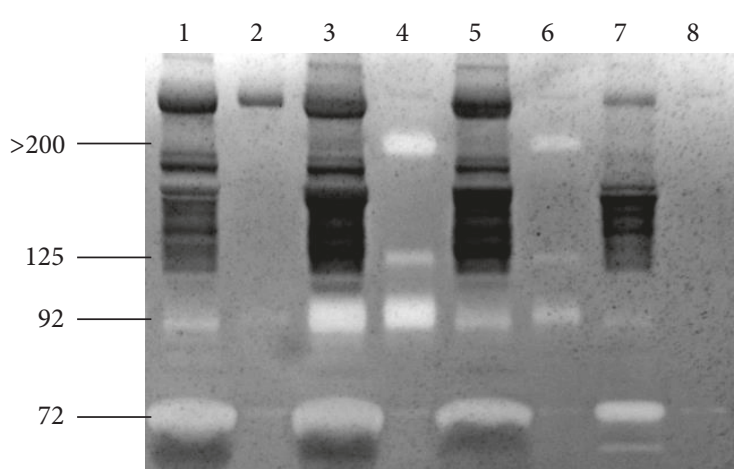

(a)

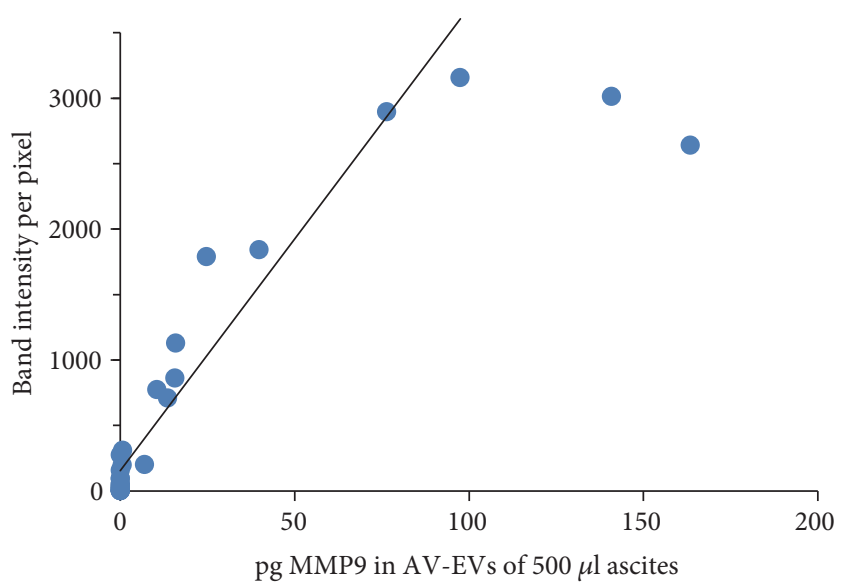

(c)

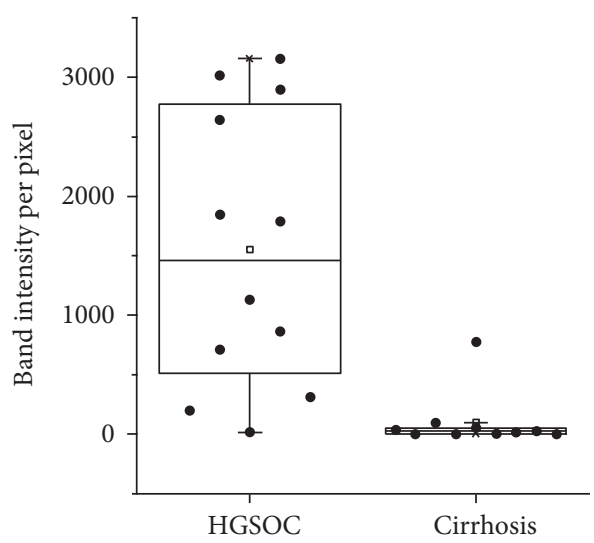

(b)

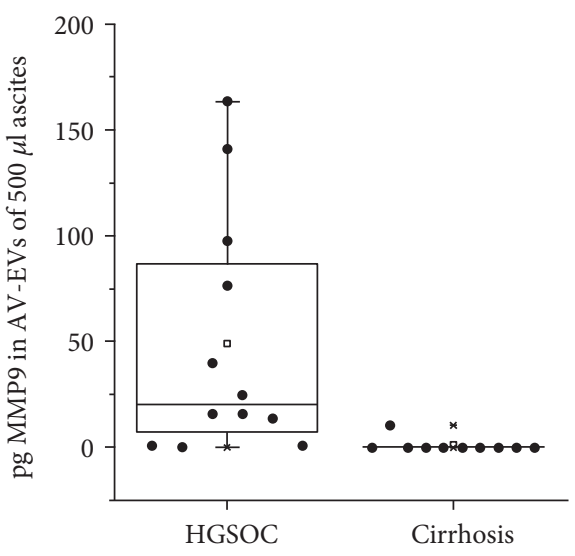

(d)

FIGURE 3: MMP9 presence and protein-level in AV-binding EVs from ascites. (a) Representative image of a zymogram to determine the presence of MMP9 in ascites samples from four individuals, where $1 \mu \mathrm{l}$ of ascites (lanes 1, 3, 5, and 7) and AV-binding EVs from $500 \mu \mathrm{l}$ ascites (lanes 2, 4, 6, and 8) were loaded. The sample in lanes 1 and 2 is from a patient with carcinosarcoma, the samples in lanes 3, 4, and 5, 6 are each from patients with HGSOC, and the sample in lanes 7 and 8 is from a patient with cirrhosis. (b) Box plot showing the difference in MMP9 band intensity analyzed by zymography in AV-EVs isolated from different ascites samples. (c) Correlation graph of MMP9-levels measured with zymography ( $y$-axis) and ELISA ( $x$-axis). (d) Box plot showing the difference in MMP9 amount analyzed by ELISA in AV-EVs isolated from different ascites samples (HGSOC = high-grade serous ovarian cancer).

further demonstrated that the enzyme was present in its inactive proform and was restricted specifically to the AVbinding EVs (Figure 2(b)). This enzyme was not present in the CTB- or STB-EVs or in any of the three EV types isolated from the portal-hypertensive ascites. In addition to pro-MMP9 at $92 \mathrm{kDa}$, we observed two higher molecular weight MMPs at $>200 \mathrm{kDa}$ and $125 \mathrm{kDa}$ in the AV-binding EVs from pooled ovarian cancer ascites. We hypothesized that the former could be multimers of MMP9 [40], while the latter could be a complex of MMP9 and neutrophil gelatinase B-associated lipocalin (NGAL) [40], which has been described in AV-binding EVs secreted by activated neutrophils [41].

To rule out any anomalies caused by the pooling of ascitic fluids, we analyzed ascites samples individually with zymography. Interestingly, we did not detect MMP9 in the $\mathrm{AV}$-EVs from the ascites of patients with carcinosarcoma or clear cell histology. Instead, MMP9 was detected in AV-binding EVs of patients with high-grade serous ovarian cancer (HGSOC) (Figure 3(a)). The analysis of the gelatinolytic band intensities revealed that MMP9levels were significantly higher in AV-EVs from HGSOC ascites compared to ascites AV-EVs from patients with cirrhosis $\left(p=6 \times 10^{-4}\right)$ (Figure $\left.3(\mathrm{~b})\right)$.

For further evaluation of this finding, the MMP9 concentration in AV-binding EVs was quantified by a sandwich ELISA. This was performed, because the detection range by zymography is very narrow. As shown in Figure 3(c), the band intensities in zymography only correlated with MMP9-levels up to $100 \mathrm{pg}$ MMP9 per $500 \mu \mathrm{l}$ ascites at Spearman's rho of $0.885\left(p=1.85 \times 10^{-9}\right)$. Based on the ELISA analysis, MMP9-levels in AV-EVs were statistically significantly higher in patients with HGSOC, when compared to patients with cirrhosis $\left(p=3 \times 10^{-4}\right)$ (Figure $3(\mathrm{~d})$ ).

To confirm that the lack of detectable MMP9 in AVbinding EVs from portal-hypertensive ascites was not due to the absence of AV-EVs, we examined ascites by the labeling of EVs with AV-conjugated gold nanoparticles and 


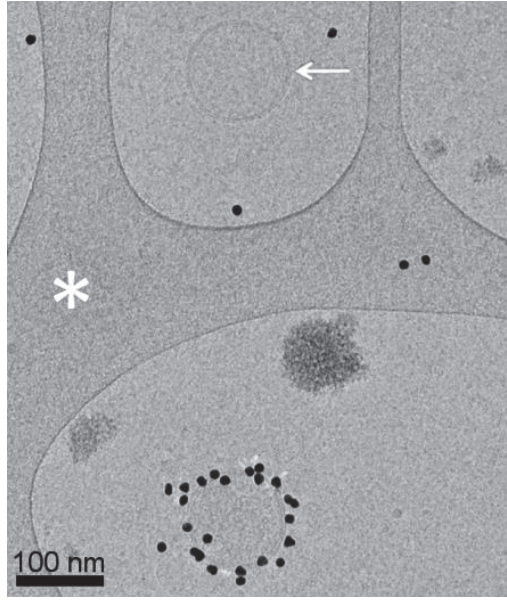

(a)

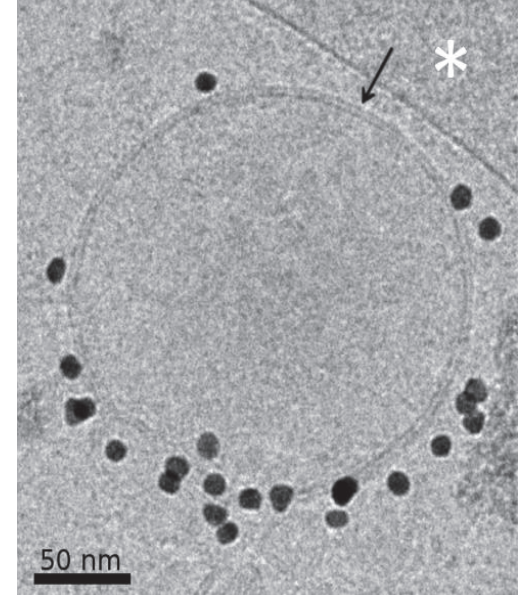

(b)

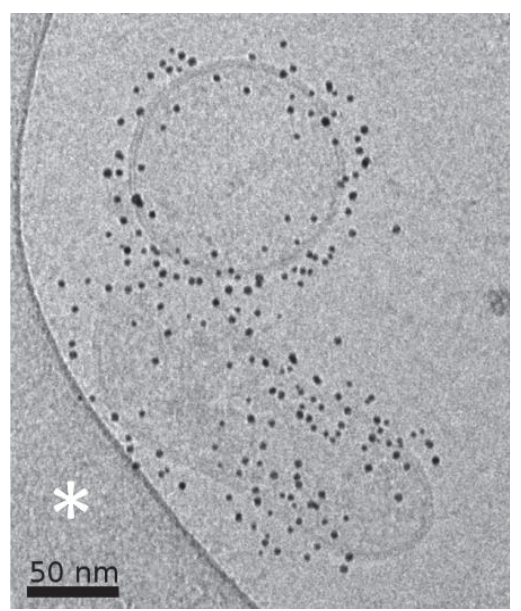

(c)

FIgURE 4: Cryo-EM images of individual EVs from the ascites of patients with HGSOC (a), carcinosarcoma (b), or liver cirrhosis (c). The samples were labeled with AV-conjugated gold nanoparticles (10 nm diameter in (a) and (b), $4 \mathrm{~nm}$ diameter in (c)). In (a), a non-AVbinding EV (white arrow) is seen, next to an AV-labeled EV. The lipid bilayer surrounding EVs is clearly resolved by cryo-EM (black arrow in (b)). The white asterisks point to areas of the carbon support film. Scale bars: $100 \mathrm{~nm}$ in (a) and $50 \mathrm{~nm}$ in (b) and (c).

imaging with cryo-EM [15]. In all of the examined samples, including those that did not have detectable MMP9 in AVEVs, both AV-binding EVs and non-AV-binding EVs were observed (Figure 4). In addition, both EV populations exhibited the characteristic lipid bilayer with a size range characteristic of EVs, that is, 50 to $500 \mathrm{~nm}$ in diameter [3].

As mentioned in the introduction, one of the greatest obstacles for the usage of EVs as biomarker in clinical applications is the lack of isolation and analysis methods that reliably and reproducibly purify and identify distinct populations of EVs, because contaminants like protein aggregates or lipoproteins that are abundantly present in body fluids are often copurified and cannot be discriminated from EVs. Consequently, the identification of distinct and clearly defined vesicle populations that harbor potential biomarkers is required for a successful clinical application. The AV-binding and MMP9-carrying EVs that were detected in HGSOC patients are candidates for utilization as ovarian cancer marker, because this EV population is defined by a combination of lipid and protein markers, which enhances the ability of specific purification and detection from complex body fluids. Even though these EVs were identified in ascites, they have potential as circulating marker, because of the constant exchange of fluids and molecules between the peritoneal cavity, the lymphatic system, and the blood stream $[42,43]$.

\section{Conclusion}

In summary, we demonstrated here a proof of concept that using membrane lipid-binding ligands to isolate different EV subpopulations from ascites fluid is an efficient method for biomarker discovery as it will enhance the signal-tonoise ratio. Specifically, by using ascites from small patient groups with HGSOC or cirrhosis and a small set of cancerassociated proteins, we observed that EV-associated MMP9 in HGSOC is predominantly localized in AV-binding EVs and that both CD59 and MMP9 in AV-binding EVs are candidate biomarkers for HGSOC. Without fractionating the EVs into subpopulations, MMP9 was unlikely to be 
identified as a candidate HGSOC biomarker as the high level of MMP9 in AV-EVs would have been obscured by the lack of MMP9 in other EVs. The utility of CD59 and MM9 in AV-binding EVs as ovarian cancer biomarker will have to be further evaluated in large patient cohort studies for their specificity and sensitivity.

\section{Conflicts of Interest}

There is no conflict of interest.

\section{Acknowledgments}

This work was supported by the Austrian Federal Ministry for Transport, Innovation and Technology (GZ BMVIT$612.166 / 0001-\mathrm{III} / \mathrm{I} 1 / 2010)$ via the International Graduate School Bio-Nano-Tech (a joint Ph.D. program of the University of Natural Resources and Life Sciences Vienna (BOKU), the Austrian Institute of Technology (AIT), and the Nanyang Technological University (NTU)) and by the Institute of Medical Biology of the Agency for Science, Technology and Research (A*STAR).

\section{References}

[1] E. Kipps, D. S. Tan, and S. B. Kaye, "Meeting the challenge of ascites in ovarian cancer: new avenues for therapy and research," Nature Reviews. Cancer, vol. 13, no. 4, pp. 273282, 2013.

[2] E. Lengyel, "Ovarian cancer development and metastasis," The American Journal of Pathology, vol. 177, no. 3, pp. 1053-1064, 2010.

[3] M. Colombo, G. Raposo, and C. Thery, "Biogenesis, secretion, and intercellular interactions of exosomes and other extracellular vesicles," Annual Review of Cell and Developmental Biology, vol. 30, pp. 255-289, 2014.

[4] M. K. Tang and A. S. Wong, "Exosomes: Emerging biomarkers and targets for ovarian cancer," Cancer Letters, vol. 367, no. 1, pp. 26-33, 2015.

[5] K. D. Dorayappan, J. J. Wallbillich, D. E. Cohn, and K. Selvendiran, "The biological significance and clinical applications of exosomes in ovarian cancer," Gynecologic Oncology, vol. 142, no. 1, pp. 199-205, 2016.

[6] P. G. Righetti, A. Castagna, F. Antonucci et al., "Proteome analysis in the clinical chemistry laboratory: myth or reality?" Clinica Chimica Acta, vol. 357, no. 2, pp. 123-139, 2005.

[7] K. W. Witwer, E. I. Buzás, L. T. Bemis et al., "Standardization of sample collection, isolation and analysis methods in extracellular vesicle research," Journal of Extracellular Vesicles, vol. 2, no. 1, p. 20360, 2013.

[8] R. Linares, S. Tan, C. Gounou, N. Arraud, and A. R. Brisson, "High-speed centrifugation induces aggregation of extracellular vesicles," Journal of Extracellular Vesicles, vol. 4, no. 1, p. 29509, 2015.

[9] Y. Yuana, J. Levels, A. Grootemaat, A. Sturk, and R. Nieuwland, "Co-isolation of extracellular vesicles and high-density lipoproteins using density gradient ultracentrifugation," Journal of Extracellular Vesicles, vol. 3, no. 1, p. 23262, 2014.

[10] B. W. Sodar, Á. Kittel, K. Pálóczi et al., "Low-density lipoprotein mimics blood plasma-derived exosomes and microvesicles during isolation and detection," Scientific Reports, vol. 6, p. 24316, 2016.

[11] A. Bobrie, M. Colombo, S. Krumeich, G. Raposo, and C. Théry, "Diverse subpopulations of vesicles secreted by different intracellular mechanisms are present in exosome preparations obtained by differential ultracentrifugation," Journal of Extracellular Vesicles, vol. 1, no. 1, p. 18397, 2012.

[12] E. Willms, H. J. Johansson, I. Mäger et al., "Cells release subpopulations of exosomes with distinct molecular and biological properties," Scientific Reports, vol. 6, p. 22519, 2016.

[13] R. C. Lai, S. S. Tan, R. W. Yeo et al., "MSC secretes at least 3 EV types each with a unique permutation of membrane lipid, protein and RNA," Journal of Extracellular Vesicles, vol. 5, p. $29828,2016$.

[14] K. Hian Tan, S. S. Tan, S. K. Sze, W. K. Lee, M. J. Ng, and S. K. Lim, "Plasma biomarker discovery in preeclampsia using a novel differential isolation technology for circulating extracellular vesicles," American Journal of Obstetrics and Gynecology, vol. 211, no. 4, pp. 380.e1-380.e13, 2014.

[15] N. Arraud, R. Linares, S. Tan et al., "Extracellular vesicles from blood plasma: determination of their morphology, size, phenotype and concentration," Journal of Thrombosis and Haemostasis, vol. 12, no. 5, pp. 614-627, 2014.

[16] N. Ahmed and K. L. Stenvers, "Getting to know ovarian cancer ascites: opportunities for targeted therapy-based translational research," Frontiers in Oncology, vol. 3, p. 256, 2013.

[17] K. Auer, A. Bachmayr-Heyda, S. Aust et al., "Peritoneal tumor spread in serous ovarian cancer-epithelial mesenchymal status and outcome," Oncotarget, vol. 6, no. 19, pp. 17261-17275, 2015.

[18] A. Bachmayr-Heyda, K. Auer, N. Sukhbaatar et al., "Small RNAs and the competing endogenous RNA network in high grade serous ovarian cancer tumor spread," Oncotarget, vol. 7, no. 26, pp. 39640-39653, 2016.

[19] K. Auer, A. Bachmayr-Heyda, N. Sukhbaatar et al., "Role of the immune system in the peritoneal tumor spread of high grade serous ovarian cancer," Oncotarget, vol. 7, no. 38, pp. 6133661354, 2016.

[20] E. S. White and A. F. Muro, "Fibronectin splice variants: understanding their multiple roles in health and disease using engineered mouse models," IUBMB Life, vol. 63, no. 7, pp. 538-546, 2011.

[21] J. N. Rybak, C. Roesli, M. Kaspar, A. Villa, and D. Neri, “The extra-domain A of fibronectin is a vascular marker of solid tumors and metastases," Cancer Research, vol. 67, no. 22, pp. 10948-10957, 2007.

[22] W. R. Jarnagin, D. C. Rockey, V. E. Koteliansky, S. S. Wang, and D. M. Bissell, "Expression of variant fibronectins in wound healing: cellular source and biological activity of the EIIIA segment in rat hepatic fibrogenesis," The Journal of Cell Biology, vol. 127, no. 6 Part 2, pp. 2037-2048, 1994.

[23] Z. Fishelson, N. Donin, S. Zell, S. Schultz, and M. Kirschfink, "Obstacles to cancer immunotherapy: expression of membrane complement regulatory proteins (mCRPs) in tumors," Molecular Immunology, vol. 40, no. 2-4, pp. 109-123, 2003.

[24] A. Clayton, C. L. Harris, J. Court, M. D. Mason, and B. P. Morgan, "Antigen-presenting cell exosomes are protected from complement-mediated lysis by expression of CD55 and CD59," European Journal of Immunology, vol. 33, no. 2, pp. 522-531, 2003. 
[25] A. A. Babiker, B. Nilsson, G. Ronquist, L. Carlsson, and K. N. Ekdahl, "Transfer of functional prostasomal CD59 of metastatic prostatic cancer cell origin protects cells against complement attack," Prostate, vol. 62, no. 2, pp. 105-114, 2005.

[26] R. Lai, R. C. Lai, R. W. Y. Yeo et al., "Mesenchymal stem cell exosomes: The future MSC-based therapy?" in Mesenchymal Stem Cell Therapy, L. G. Chase and M. C. Vemuri, Eds., pp. 39-62, Springer Science and Business Media, New York, 2013.

[27] L. Bjorge, J. Hakulinen, O. K. Vintermyr et al., "Ascitic complement system in ovarian cancer," British Journal of Cancer, vol. 92, no. 5, pp. 895-905, 2005.

[28] H. Im, H. Shao, Y. I. Park et al., "Label-free detection and molecular profiling of exosomes with a nano-plasmonic sensor," Nature Biotechnology, vol. 32, no. 5, pp. 490-5, 2014.

[29] D. D. Taylor and C. Gercel-Taylor, "MicroRNA signatures of tumor-derived exosomes as diagnostic biomarkers of ovarian cancer," Gynecologic Oncology, vol. 110, no. 1, pp. 13-21, 2008.

[30] S. Runz, S. Keller, C. Rupp et al., "Malignant ascites-derived exosomes of ovarian carcinoma patients contain CD24 and EpCAM," Gynecologic Oncology, vol. 107, no. 3, pp. 563-571, 2007.

[31] J. Z. Press, M. Reyes, S. J. Pitteri et al., "Microparticles from ovarian carcinomas are shed into ascites and promote cell migration," International Journal of Gynecological Cancer, vol. 22, no. 4, pp. 546-552, 2012.

[32] Z. Zhao, Y. Yang, Y. Zeng, and M. He, "A microfluidic ExoSearch chip for multiplexed exosome detection towards blood-based ovarian cancer diagnosis," Lab on a Chip, vol. 16, no. 3, pp. 489-496, 2016.

[33] B. T. MacDonald, K. Tamai, and X. He, "Wnt/beta-catenin signaling: components, mechanisms, and diseases," Developmental Cell, vol. 17, no. 1, pp. 9-26, 2009.

[34] S. Dovrat, M. Caspi, A. Zilberberg et al., "14-3-3 and betacatenin are secreted on extracellular vesicles to activate the oncogenic Wnt pathway," Molecular Oncology, vol. 8, no. 5, pp. 894-911, 2014.

[35] T. N. Young, G. C. Rodriguez, A. R. Rinehart, R. C. Bast Jr, S. V. Pizzo, and M. S. Stack, "Characterization of gelatinases linked to extracellular matrix invasion in ovarian adenocarcinoma: purification of matrix metalloproteinase 2," Gynecologic Oncology, vol. 62, no. 1, pp. 89-99, 1996.

[36] D. A. Fishman, L. M. Bafetti, S. Banionis, A. S. Kearns, K. Chilukuri, and M. S. Stack, "Production of extracellular matrix-degrading proteinases by primary cultures of human epithelial ovarian carcinoma cells," Cancer, vol. 80, no. 8, pp. 1457-1463, 1997.

[37] L. Manenti, P. Paganoni, I. Floriani et al., "Expression levels of vascular endothelial growth factor, matrix metalloproteinases 2 and 9 and tissue inhibitor of metalloproteinases 1 and 2 in the plasma of patients with ovarian carcinoma," European Journal of Cancer, vol. 39, no. 13, pp. 1948-1956, 2003.

[38] J. Cathcart, A. Pulkoski-Gross, and J. Cao, "Targeting matrix metalloproteinases in cancer: bringing new life to old ideas," Genes and Diseases, vol. 2, no. 1, pp. 26-34, 2015.

[39] L. E. Graves, E. V. Ariztia, J. R. Navari, H. J. Matzel, M. S. Stack, and D. A. Fishman, "Proinvasive properties of ovarian cancer ascites-derived membrane vesicles," Cancer Research, vol. 64, no. 19, pp. 7045-7049, 2004.

[40] P. E. Van den Steen, B. Dubois, I. Nelissen, P. M. Rudd, R. A. Dwek, and G. Opdenakker, "Biochemistry and molecular biology of gelatinase B or matrix metalloproteinase-9 (MMP-9),"
Critical Reviews in Biochemistry and Molecular Biology, vol. 37, no. 6, pp. 375-536, 2002.

[41] O. Gasser, C. Hess, S. Miot, C. Deon, J. C. Sanchez, and J. A. Schifferli, "Characterisation and properties of ectosomes released by human polymorphonuclear neutrophils," Experimental Cell Research, vol. 285, no. 2, pp. 243-257, 2003.

[42] K. F. Mc, W. G. Wilt Jr, R. E. Hyatt, and G. H. Whipple, "The circulation of ascitic fluid; interchange of plasma and ascitic fluid protein as studied by means of C14-labeled lysine in dogs with constriction of the vena cava," The Journal of Experimental Medicine, vol. 91, no. 2, pp. 115-122, 1950.

[43] D. S. Tan, R. Agarwal, and S. B. Kaye, "Mechanisms of transcoelomic metastasis in ovarian cancer," The Lancet Oncology, vol. 7, no. 11, pp. 925-934, 2006. 


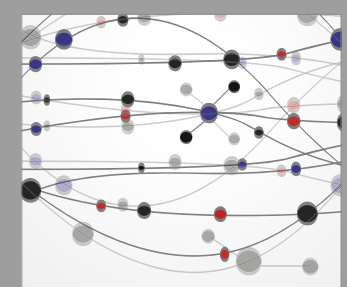

The Scientific World Journal
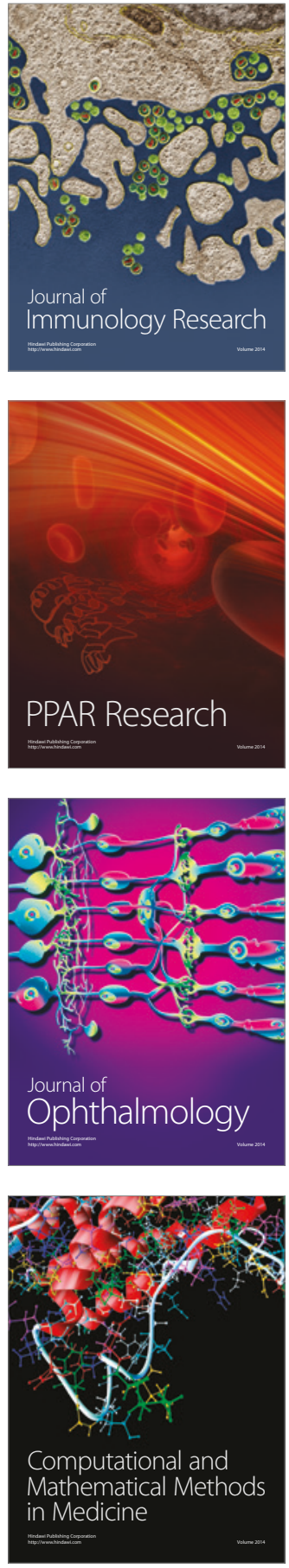

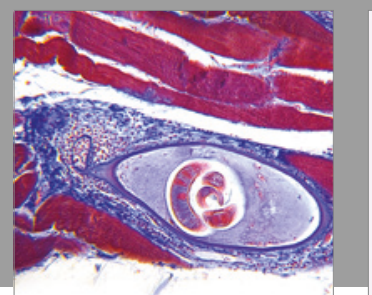

Gastroenterology Research and Practice
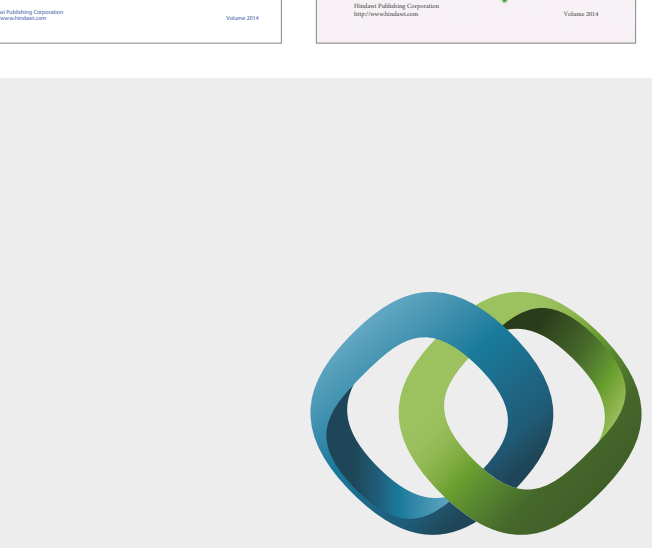

\section{Hindawi}

Submit your manuscripts at

https://www.hindawi.com
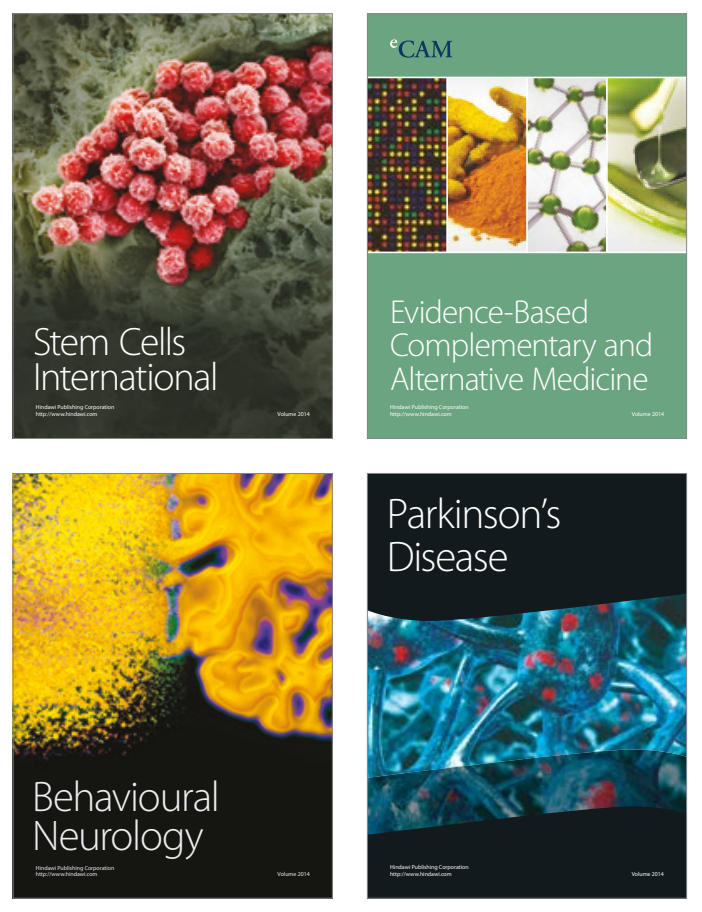
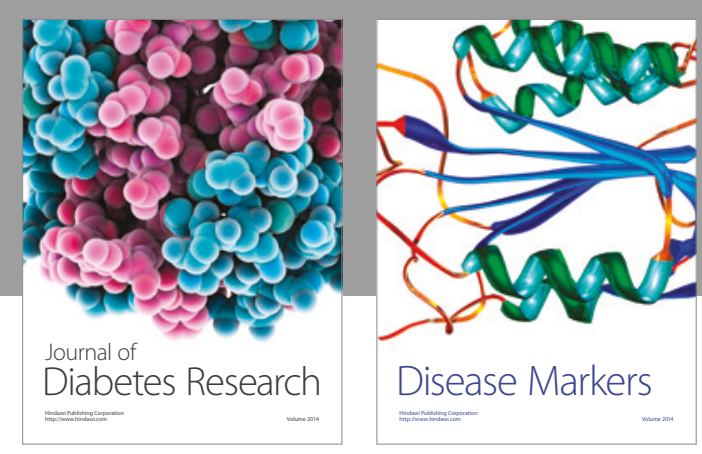

Disease Markers
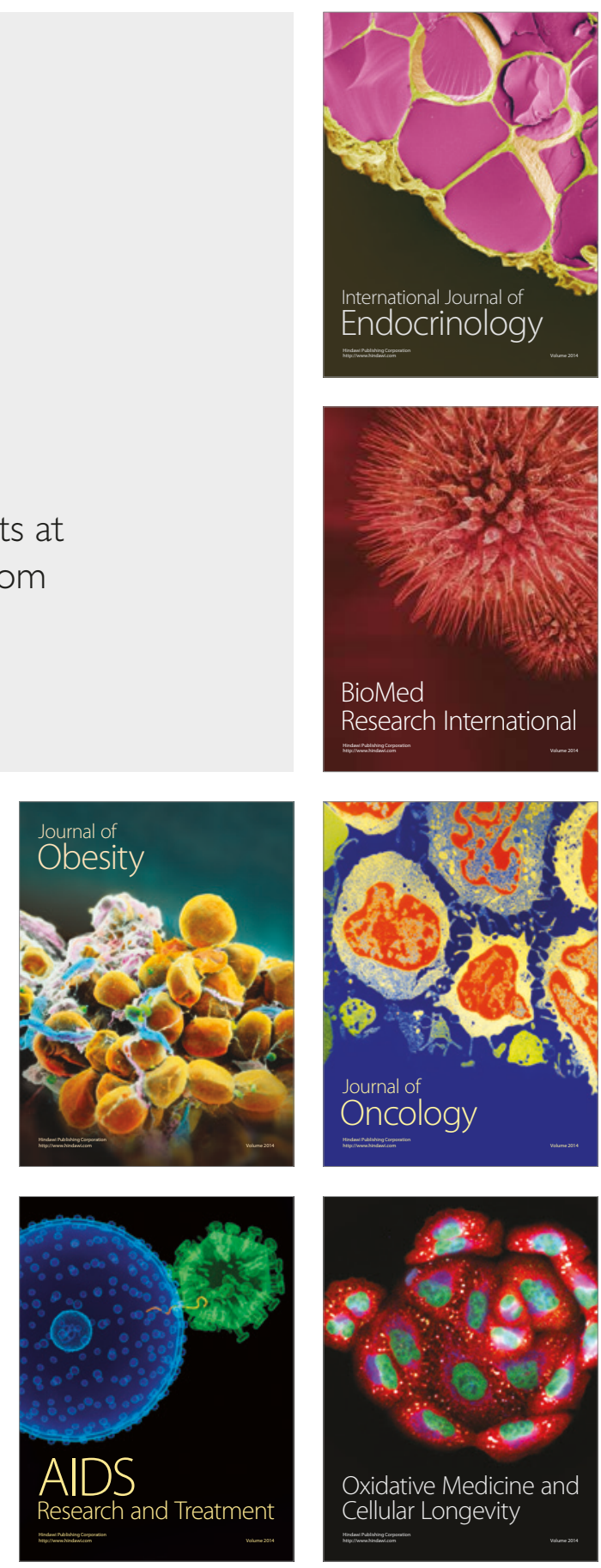